\title{
A Review of Diamond-Blackfan Anemia: Current Evidence on Involved Genes and Treatment Modalities
}

\author{
Anshika Tyagi $^{1}$, Apurv Gupta ${ }^{1}$, Anirban Dutta ${ }^{2}$, Pooja Potluri ${ }^{3}$, Badie Batti $^{4}$ \\ 1. Medicine, Maulana Azad Medical College, New Delhi, IND 2. Medicine, Dr. NMB Baruah Nursing Home, Nalbari, IND \\ 3. Medicine, Jawaharlal Nehru Medical College, Belgaum, IND 4. Medicine, Alkindi Teaching Hospital, Baghdad, IRQ
}

Corresponding author: Anshika Tyagi, tyagianshika1996@gmail.com

\begin{abstract}
Diamond-Blackfan anemia (DBA) is a congenital cause of bone marrow failure predominantly involving the erythroid cell line, with occasional impact on other cell lines. In the vast majority of cases, it is diagnosed by one year of age. We looked at the existing literature on the disease presentation along with established as well as upcoming treatment options. Numerous genes have been identified and extensively studied in the context of their part in the pathogenesis of DBA. Treatment revolves around the use of steroids and regular blood transfusions, with hematopoietic stem cell transplantation reserved for steroid-resistant cases. Newer modalities such as gene therapy, l-leucine, sotatercept, trifluoperazine, SMER28, and danazol are also concisely discussed. The purpose of this article is to review the previous literature on DBA and weigh the role of newer therapeutic agents.
\end{abstract}

Categories: Pathology, Pediatrics, Hematology

Keywords: anemia, inherited bone marrow failure, ribosomopathy

\section{Introduction And Background}

Diamond-Blackfan anemia (DBA) is a congenital bone marrow failure syndrome that chiefly affects the erythroid precursors. It presents mostly as severe anemia in infants, along with craniofacial anomalies, poor growth, and various abnormalities of the limb and viscera. The diagnostic investigation is bone marrow smear, which shows a decreased number of erythroid precursors but a normal cell population of myeloid and megakaryocytic lineage [1]. The hematological profile is that of normochromic normocytic or macrocytic anemia [2]. Another evidence of an inherent defect in the pathway of erythropoiesis can be found in the recovery of hemoglobin levels after bone marrow transplantation [3]. Some studies have suggested that not only the erythroid lineage but, in some patients, myeloid and lymphoid precursors are also affected, thus leading to neutropenia and lymphopenia [4].

Received 08/11/2020

Review began 08/13/2020 Review ended 08/18/2020 Published 08/25/2020

() Copyright 2020

Tyagi et al. This is an open access article distributed under the terms of the Creative Commons Attribution License CC-BY 4.0., which permits unrestricted use, distribution, and reproduction in any medium, provided the original author and source are credited.
Since the time DBA was first noticed, we have come a long way in our understanding of this disease. It was first recognized by Josephs in 1936 [5], but a detailed description was provided by Diamond and Blackfan in 1938 [6]. Several studies have been published in the 84 years we have known about DBA, each with a different focus. The objective of this article is to examine the contributions made by these studies to the development of our knowledge of the disease while also highlighting the potential areas of future research concerning the treatment of DBA.

\section{Review}

\section{Epidemiology}

DBA registries were set up in North America and the United Kingdom to elucidate the epidemiology, pathophysiology, and prognosis of the condition. The data from the UK registry suggested an annual incidence of five per million live births after a retrospective analysis of 80 cases followed over 20 years (1975-1994) [7]. The prevalence is independent of the gender and ethnicity of the population. The majority of the cases are sporadic except a few that are familial. In a study by Orfali et al., data from 60 families for the evaluation of genetics and familial patterns was collected [8]. Nine out of 60 patients were known to have a family history of DBA. Five of those nine patients with a known family history were elucidated in this study to have autosomal dominant mode of inheritance. Hematological abnormalities were found in family members of 16 out of those 51 patients who were initially thought to be sporadic cases of DBA. Out of 51 cases with no known family history, 35 showed no hematological abnormalities in their family members, confirming their sporadic origin. To summarize, DBA in $58 \%$ of the evaluated 60 families was sporadic [8].

\section{Etiology}

Various genetic mutations are responsible for the pathogenesis of DBA (Table 1). The list includes mutations in the genes encoding ribosomal proteins of small and large ribosomal subunits. Common genes and the 
frequency of their involvement are as follows: RPS19 (25\%) [9], RPS24 (approximately 2\%) [10], RPS17 [11], RPL5 and RPL11 (6.6\% and 4.8\%, respectively) [12], and RPS10 and RPS26 (2.6\% and 6.4\%, respectively) [13].

\begin{tabular}{|l|l|}
\hline Ribosomal protein gene & Year of discovery \\
\hline RPS19 & 1999 \\
RPS24 & 2006 \\
RPS17 & 2007 \\
RPL5 and RPL11 & 2008 \\
RPS10 and RPS26 & 2010 \\
\hline
\end{tabular}

TABLE 1: Chronology of discovery of ribosomal protein gene mutations in DBA

DBA, Diamond-Blackfan anemia

\section{RPS24 Gene}

A genome-wide linkage analysis was performed in a study that had enrolled 215 families with DBA phenotype [10]. A region on chromosome 10 was identified as the cause of the hypoproliferation of erythroid precursors. Further sequencing of this region revealed a nonsense mutation $(316 \mathrm{C}->\mathrm{T})$ in the gene for ribosomal protein synthesis. This nonsense mutation is conducive to the formation of a truncated ribosomal protein S24 [10].

\section{RPL5 and RPL11}

These genes are present on chromosome 1, and their protein products form the large subunits of ribosomes. The involvement of this chromosome in pathogenesis was first described by Heyn et al. in 1974 when they observed pericentric inversion of the first chromosome in a DBA patient with cleft lip and palate [14]. Bearing this in mind, five ribosomal protein genes on chromosome 1 were screened in a study with 196 families, and numerous alterations in the sequences of coding regions of RPL5 and RPL11 were noticed [12]. RPL5 and RPL11 gene mutations were associated with a significant frequency ( $70 \%$ and $67 \%$, respectively) of physical malformations as compared to other gene mutations in DBA (46\% in RPS19 mutations) [12].

\section{RPS10 and RPS26}

A study conducted in 2010 with the participation of 117 DBA families aimed to accomplish a large extent screen of ribosomal protein mutations in a cohort of DBA patients [13]. Five out of 117 probands had mutations in the RPS10 gene sequence on chromosome 10, and 12 out of 117 probands had mutations in the RPS26 gene sequence on chromosome 12 [13]. RPS10 and RPS26 genes form proteins of small subunits of ribosomes. RPS10 genes were maximally noted to have undergone nonsense mutation and RPS26 predominantly harbored mutations in the translation initiation codon [13].

\section{Pathogenesis}

The role of ribosomal protein gene mutations has been studied extensively in the context of RPS19 gene mutation. The RPS19 gene is located on the 19th chromosome. Its protein product is required for the development of $18 \mathrm{~S}$ rRNA and the integration of pre-40S particles during ribosome biogenesis [15,16]. Flygare et al. demonstrated the effect of reduced expression of RPS19 on ribosomal maturation in the erythropoietic cell line of TF-1 [15], whereas Choesmel et al. demonstrated the same effect on the HeLa cell line [16]. This defect in the synthesis of ribosomes affects the cells, which undergo rapid proliferation and differentiation like erythroblasts [16]. Poor ribosomal biogenesis causes increased nucleolar stress, which further leads to enhanced binding of certain ribosomal proteins to p53 inhibitor MDM2 (murine double minute 2) [17]. This binding results in decreased inhibition of $\mathrm{p} 53$ by MDM2. Accumulation of $\mathrm{p} 53$ protein causes cell cycle arrest and apoptosis in the erythroid lineage. To explain the exclusive loss of erythroid precursors in DBA, the levels of $\mathrm{p} 53$ and $\mathrm{p} 21$ protein in erythroid differentiated cells were compared with those in myeloid and megakaryocytic cells. It was noticed that the levels were significantly higher in erythroid cells, thus explaining the lineage specificity of DBA [18]. It is understood now that mutations in ribosomal protein genes besides RPS19 can also bring about deficiency in the biogenesis of ribosomes. For example, RPL26 protein product too is instrumental in the maturation of 18s rRNA and is a regulator of p53 just like RPS19 [19]. Thus, its deficiency has similar effects as RPS19 deficiency.

A study in 2012 had suggested one more mechanism behind erythroid aplasia through RPS19 mutations - an anomaly in the translation of certain mRNA transcripts directly or indirectly responsible for the growth of 
red blood cells [20]. Rarely, DBA patients may exhibit mutations in GATA1 protein, which is a transcription factor involved in erythroid differentiation of cell lines [21]. This study indicated the role of transcription factors in the pathogenesis of DBA, which should be further explored.

\section{Clinical features}

An analysis of DBA's clinical features was performed by Willig et al. in 1999 using the French registry of DBA patients [22]. A total of 229 patients were followed for 13 years. The diagnostic criterion used in this study was the presence of less than $5 \%$ of erythroid precursors in the bone marrow with a background of normal population of other cells. It was observed that $98.7 \%$ of cases were diagnosed by the age of five years, with $88 \%$ of them being diagnosed by one year of age [22]. Around $40 \%$ cases of DBA have physical malformations [7]. Detailed incidences of various physical malformations were described in the study by Willig et al. [22]. The highest recorded incidence was of head malformations (20.5\%) followed by eye defects. The least incidence was that of neck deformities [22]. The head malformations included microcephaly, flat nose, cleft palate, microretrognathia, high arch palate, low set ears, anomalies of the ears, low hairline, Pierre-Robin's syndrome, and others [22].

\section{Complications}

The association between DBA and increased susceptibility to cancer had been qualitatively described in many case reports, but the first quantitative analysis of the exact increase in risk was provided by Vlachos et al. [23]. This study described cancer predilection in terms of relative risk and cumulative incidence. The relative risk of DBA patients developing cancer was 5.4 [23]. The gross cumulative incidence of all cancers, except myelodysplastic syndrome, in DBA patients by 46 years of age was $22 \%$ [23]. In 2018, Vlachos et al. released another analysis that was enhanced with additional six-year information. The relative risk of cancer as calculated in this report was 4.8 and the cumulative incidence of all the statistically evaluable cancers was 13.7\% [24]. The difference may simply be a result of an improved database. The common malignancies in DBA according to the report were colon carcinoma and osteogenic sarcoma [24].

Other complications are related to the treatment of DBA. Long-term use of steroids has its own set of problems such as Cushing's syndrome, steroid-induced cataract, and others. Chronic red cell transfusion lands the patient in iron overload and its consequent troubles such as hemochromatosis. Endocrine complications in DBA have also been studied [25]. The highest percentage was determined to be of adrenal insufficiency and hypogonadism [25]. The frequency of endocrine dysfunction was more in patients who were transfusion-dependent as compared to those on steroids [25].

\section{Diagnosis}

The scaffolding on which the current diagnostic criteria are based remains the one described by Diamond et al. in their 1976 review [26]. These classical criteria include macrocytic anemia, presentation before the age of one year, normal counts of leukocytes and platelets, reticulocytopenia, and marrow examination revealing depleted red cell precursors [27]. Supporting criteria include major criteria, which are positive family history and the discovery of a pathogenic mutation, and minor criteria, which are congenital anomalies, raised levels of erythrocyte adenosine deaminase (ADA), which are raised in $85 \%$ of cases, increased hemoglobin F, and exclusion of other syndromes of inherited bone marrow failure ( [IBMF] Fanconi's syndrome, Shwachman-Diamond's syndrome) [27].

The investigations ordered are mostly to rule out the many different causes of infantile anemia, for example, $\mathrm{ABO}$ and $\mathrm{Rh}$ incompatibility, drugs and toxins, HIV infection, nutritional deficiencies, and transient erythroblastopenia of childhood. This list is not exhaustive. The presence of specific features such as bone marrow smear picture, associated physical abnormalities, elevated erythrocytic ADA, elevated HbF (fetal hemoglobin), and positive family history guides us toward a diagnosis of IBMFs. The differentiation between the different IBMF syndromes is largely reliant on the presence of genetic mutations specific to each condition.

\section{Treatment}

The historical and most utilized treatment in DBA is corticosteroid. The literature suggests that $80 \%$ of patients show improvement after the first course of steroids, but the remaining $20 \%$ do not (steroidresistant) and hence are started on red cell transfusion. A follow-up of the patients registered in a DBA registry disclosed that $37 \%$ were maintained on steroids and $31 \%$ on chronic red cell transfusion [28]. Of the transfusion-dependent patients, $22 \%$ were steroid responsive to start with but stopped responding over time and hence had to be started on transfusion [28]. Hematopoietic stem cell transplantation (HSCT) is reserved for patients who have developed transfusion dependence. The advantage of HSCT is that it provides a permanent cure and abrogates the need for regular transfusions. The limitations, however, are that the procedure is associated with graft versus host disease and infections secondary to the use of immunosuppression. The first HSCT for DBA was performed by August et al. in 1976 [29]. A study revealed that HSCT of HLA (human leukocyte antigen)-matched donors yielded better outcomes as compared to alternative donors [30]. The outcomes were also dependent on the pre-transplantation status of iron overload in the patient's body. The lower the load, the better the survival rate [30]. The development of 


\section{Gene Therapy}

The success of gene therapy was demonstrated in a study in which lymphoblastoid cells were collected from patients with DBA [31]. These cells with RPS19 mutation showed retarded maturation of pre-rRNA, decreased protein formation due to poor ribosomal synthesis, and ribosomal stress manifested as p53 activation. After they were treated with viral vectors carrying the RPS19 gene, these cells recovered their protein synthesis and levels of p53 also dropped [31]. Another study reported the efficacy of gene therapy by exhibiting successful treatment of RPS19-deficient DBA mouse models following gene transfer [32].

Stimulation of RPS19 gene expression in RPS19-deficient cells by the transfer of lentiviral vector with cDNA of elongation factor $1 \alpha$ short (EFS) promoter has also been documented [33]. This provides further direction to investigation in the field of gene therapy as a therapeutic measure.

\section{L-Leucine}

Leucine is an essential amino acid and is a regulator of cellular protein synthesis through the mammalian target of rapamycin (mTOR) pathway [34]. The use of L- leucine in the improvement of erythropoiesis in DBA patients was explored by Pospisilova et al. in 2007 [35]. This principle was further proven by various studies reporting improvement in zebrafish models of DBA and in DBA patients [36,37]. A study of the role of amino acid leucine in the treatment of transfusion-dependent DBA is ongoing under ClinicalTrials.gov (NCT01362595) [38].

\section{Sotatercept (ACE-011)}

It is a human dimeric fusion protein consisting of an extracellular domain of activin type IIA receptor and Fc domain of human IgG1 [39]. Activin belongs to the transforming growth factor family. It was first purified from porcine ovarian follicular fluid and was demonstrated to increase the secretion of follicle-stimulating hormone from cultured anterior pituitary cells [40]. The participation of activin A in murine erythropoiesis was confirmed by Shiozaki et al. [41] and it came to be known as an erythroid differentiation factor. Sotatercept has documented efficacy in the improvement of chemotherapy-induced anemia [42]. Bearing in mind all this evidence of the potency of ACE-011, studies were conducted to explore its use in the management of DBA. Zebrafish models of DBA, in which the RPL11 ribosomal protein gene was silenced, showed enhanced erythropoiesis following treatment with RAP-011 (a murine analog of ACE-011) [43]. Therefore, this compound shows promise and is currently being evaluated in a clinical trial for DBA registered under ClinicalTrials.gov (NCT01464164) [44].

\section{Trifluoperazine}

It is a calmodulin inhibitor (used as an antipsychotic) that has been proven to be involved in promoting erythroid differentiation in many studies. Calmodulin inhibitors showed efficacy in improving pathogenic phenotypes in zebrafish embryos deficient in RPS29, human cancer cell lines deficient in RPS19, and human cord blood-derived CD34+ cells deficient in ribosomal proteins [45]. The mechanism has been interpreted as follows: trifluoperazine inhibits calmodulin-dependent kinases, which stabilize p 53 by phosphorylating it [46].

\section{SMER28}

SMER28 is a molecule that is involved in the modulation of autophagy. It was investigated as a therapeutic option in DBA. A drug screen was conducted on induced pluripotent stem cells from DBA patients (reprogrammed fibroblasts from patients with inactivating RPS19 and RPL5 mutations) and SMER28 was identified as a compound that stimulated erythropoiesis [47]. Further studies in this area should be supported.

\section{Danazol}

A case was reported in which use of danazol (synthetic steroid) resulted in the resolution of anemia in a steroid unresponsive DBA patient [48]. No clinical trials aimed at analyzing the therapeutic role of danazol in DBA have been conducted yet and should be considered in the future.

\section{Conclusions}

DBA is an inherited syndrome involving the erythroid cell line. Various gene mutations such as RPS19 and RPS26 have been described in detail in the etiopathogenesis of DBA. The cornerstone of treatment is still corticosteroid, with regular blood transfusions and HSCT reserved for more severe and steroid-resistant cases. Current evidence proves that gene therapy has shown success in mice. L-leucine and sotatercept are presently under trial, and we will soon see some developments. For future research, clinical trials with SMER28 and danazol should be considered. To conclude, with the discovery of detailed pathogenesis of DBA and responsible genes in DBA, new doors to treatment have opened, and we can expect some inclusions in the standard treatment protocol. 


\section{Additional Information \\ Disclosures}

Conflicts of interest: In compliance with the ICMJE uniform disclosure form, all authors declare the following: Payment/services info: All authors have declared that no financial support was received from any organization for the submitted work. Financial relationships: All authors have declared that they have no financial relationships at present or within the previous three years with any organizations that might have an interest in the submitted work. Other relationships: All authors have declared that there are no other relationships or activities that could appear to have influenced the submitted work.

\section{References}

1. Chirnomas SD, Kupfer GM: The inherited bone marrow failure syndromes . Pediatr Clin North Am. 2013, 60:1291-1310. 10.1016/j.pcl.2013.09.007

2. Halperin DS, Freedman MH: Diamond-Blackfan anemia: etiology, pathophysiology, and treatment . Am J Pediatr Hematol Oncol. 1989, 11:380-394.

3. Lenarsky C, Weinberg K, Guinan E, et al.: Bone marrow transplantation for constitutional pure red cell aplasia. Blood. 1988, 71:226-229. 10.1182/blood.V71.1.226.226

4. Giri N, Kang E, Tisdale JF, et al.: Clinical and laboratory evidence for a trilineage haematopoietic defect in patients with refractory Diamond-Blackfan anaemia. Br J Haematol. 2000, 108:167-175. 10.1046/j.13652141.2000.01796.x

5. Josephs HW: Anaemia of infancy and early childhood. Medicine. 1936, 15:307-451.

6. Diamond LK: Hypoplastic anemia. Am J Dis Child. 1938, 56:464.

7. Ball SE, McGuckin CP, Jenkins G, Gordon-Smith EC: Diamond-Blackfan anaemia in the U.K: analysis of 80 cases from a 20-year birth cohort. Br J Haematol. 1996, 94:645-653. https://doi.org/10.1046/j.13652141.1996.d01-1839.x

8. Orfali KA, Ohene-Abuakwa Y, Ball SE: Diamond Blackfan anaemia in the UK: clinical and genetic heterogeneity [published correction appears in. Br J Haematol. 2005, 128:243-252. 10.1111/j.13652141.2004.04890.x

9. Draptchinskaia N, Gustavsson P, Andersson B, et al.: The gene encoding ribosomal protein S19 is mutated in Diamond-Blackfan anaemia. Nat Genet. 1999, 21:169-175. 10.1038/5951

10. Gazda HT, Grabowska A, Merida-Long LB, et al.: Ribosomal protein S24 gene is mutated in DiamondBlackfan anemia. Am J Hum Genet. 2006, 79:1110-1118. 10.1086/510020

11. Cmejla R, Cmejlova J, Handrkova H, Petrak J, Pospisilova D: Ribosomal protein S17 gene (RPS17) is mutated in Diamond-Blackfan anemia. Hum Mutat. 2007, 28:1178-1182. 10.1002/humu.20608

12. Gazda HT, Sheen MR, Vlachos A, et al.: Ribosomal protein L5 and L11 mutations are associated with cleft palate and abnormal thumbs in Diamond-Blackfan anemia patients. Am J Hum Genet. 2008, 83:769-780. 10.1016/j.ajhg.2008.11.004

13. Doherty L, Sheen MR, Vlachos A, et al.: Ribosomal protein genes RPS10 and RPS26 are commonly mutated in Diamond-Blackfan anemia. Am J Hum Genet. 2010, 86:222-228. 10.1016/j.ajhg.2009.12.015

14. Heyn R, Kurczynski E, Schmickel R: The association of Blackfan-Diamond syndrome, physical abnormalities, and an abnormality of chromosome 1. J Pediatr. 1974, 85:531-533. 10.1016/S00223476(74)80464-6

15. Flygare J, Aspesi A, Bailey JC, Miyake K, Caffrey JM, Karlsson S, Ellis SR: Human RPS19, the gene mutated in Diamond-Blackfan anemia, encodes a ribosomal protein required for the maturation of $40 \mathrm{~S}$ ribosomal subunits. Blood. 2007, 109:980-986. 10.1182/blood-2006-07-038232

16. Choesmel V, Bacqueville D, Rouquette J, et al.: Impaired ribosome biogenesis in Diamond-Blackfan anemia. Blood. 2007, 109:1275-1283. 10.1182/blood-2006-07-038372

17. Lindstrom MS, Deisenroth C, Zhang Y: Putting a finger on growth surveillance: insight into MDM2 zinc finger-ribosomal protein interactions. Cell Cycle. 2007, 6:434-437. 10.4161/cc.6.4.3861

18. Dutt S, Narla A, Lin K, et al.: Haploinsufficiency for ribosomal protein genes causes selective activation of p53 in human erythroid progenitor cells. Blood. 2011, 117:2567-2576. 10.1182/blood-2010-07-295238

19. Gazda HT, Preti M, Sheen MR, et al.: Frameshift mutation in p53 regulator RPL26 is associated with multiple physical abnormalities and a specific pre-ribosomal RNA processing defect in Diamond-Blackfan anemia. Hum Mutat. 2012, 33:1037-1044. 10.1002/humu.22081

20. Horos R, Ijspeert H, Pospisilova D, et al.: Ribosomal deficiencies in Diamond-Blackfan anemia impair translation of transcripts essential for differentiation of murine and human erythroblasts. Blood. 2012, 119:262-272. 10.1182/blood-2011-06-358200

21. Sankaran VG, Ghazvinian R, Do R, et al.: Exome sequencing identifies GATA1 mutations resulting in Diamond-Blackfan anemia. J Clin Invest. 2012, 122:2439-2443. 10.1172/jci63597

22. Willig TN, Niemeyer CM, Leblanc T, et al.: Identification of New prognosis factors from the clinical and epidemiologic analysis of a registry of 229 Diamond-Blackfan anemia patients. Pediatr Res. 1999, 46:553561. 10.1203/00006450-199911000-00011

23. Vlachos A, Rosenberg PS, Atsidaftos E, Alter BP, Lipton JM: Incidence of neoplasia in Diamond Blackfan anemia: a report from the Diamond Blackfan Anemia Registry. Blood. 2012, 119:3815-3819. 10.1182/blood2011-08-375972

24. Vlachos A, Rosenberg PS, Atsidaftos E, et al.: Increased risk of colon cancer and osteogenic sarcoma in Diamond -Blackfan anemia. Blood. 2018, 132:2205-2208. 10.1182/blood-2018-05-848937

25. Lahoti A, Harris YT, Speiser PW, Atsidaftos E, Lipton JM, Vlachos A: Endocrine dysfunction in DiamondBlackfan anemia (DBA): a report from the DBA Registry (DBAR). Pediatr Blood Cancer. 2016, 63:306-312. $10.1002 / \mathrm{pbc} .25780$

26. Diamond LK, Wang WC, Alter BP: Congenital hypoplastic anemia. Adv Pediatr. 1976, 22:349-378.

27. Vlachos A, Ball S, Dahl N, et al.: Diagnosing and treating Diamond Blackfan anaemia: results of an 
international clinical consensus conference. Br J Haematol. 2008, 142:859-876. 10.1111/j.13652141.2008.07269.x

28. Lipton JM, Ellis SR: Diamond-Blackfan anemia: diagnosis, treatment, and molecular pathogenesis . Hematol Oncol Clin North Am. 2009, 23:261-282. 10.1016/j.hoc.2009.01.004

29. August CS, King E, Githens JH, McIntosh K, Humbert JR, Greensheer A, Johnson RB: Establishment of erythropoiesis following bone marrow transplantation in a patient with congenital hypoplastic anemia (Diamond-Blackfan syndrome). Blood. 1976, 48:491-498. 10.1182/blood.V48.4.491.491

30. Vlachos A, Federman N, Reyes-Haley C, Abramson J, Lipton JM: Hematopoietic stem cell transplantation for Diamond Blackfan anemia: a report from the Diamond Blackfan Anemia Registry. Bone Marrow Transplant. 2001, 27:381-386. 10.1038/sj.bmt.1702784

31. Aspesi A, Monteleone V, Betti M, et al.: Lymphoblastoid cell lines from Diamond Blackfan anaemia patients exhibit a full ribosomal stress phenotype that is rescued by gene therapy. Sci Rep. 2017, 7:12010. 10.1038/s41598-017-12307-5

32. Jaako P, Debnath S, Olsson K, et al.: Gene therapy cures the anemia and lethal bone marrow failure in a mouse model of RPS19-deficient Diamond-Blackfan anemia. Haematologica. 2014, 99:1792-1798. 10.3324/haematol.2014.111195

33. Debnath S, Jaako P, Siva K, et al.: Lentiviral Vectors with cellular promoters correct anemia and lethal bone marrow failure in a mouse model for Diamond-Blackfan anemia. Mol Ther. 2017, 25:1805-1814. 10.1016/j.ymthe.2017.04.002

34. Suryawan A, Jeyapalan AS, Orellana RA, Wilson FA, Nguyen HV, Davis TA: Leucine stimulates protein synthesis in skeletal muscle of neonatal pigs by enhancing mTORC1 activation. Am J Physiol Endocrinol Metab. 2008, 295:868-875. 10.1152/ajpendo.90314.2008

35. Pospisilova D, Cmejlova J, Hak J, Adam T, Cmejla R: Successful treatment of a Diamond-Blackfan anemia patient with amino acid leucine. Haematologica. 2007, 92:66-67. 10.3324/haematol.11498

36. Payne EM, Virgilio M, Narla A, et al.: L-Leucine improves the anemia and developmental defects associated with Diamond-Blackfan anemia and del(5q) MDS by activating the mTOR pathway. Blood. 2012,120, 22142224. 10.1182/blood-2011-10-382986

37. Vlachos A, Atsidaftos E, Muir E, et al.: Leucine for the treatment of transfusion dependence in patients with Diamond Blackfan anemia. Blood. 2018, 132:755. 10.1182/blood-2018-99-113570

38. Pilot phase I/II study of amino acid leucine in treatment of patients with transfusion-dependent Diamond Blackfan anemia (LeucineDBA). Accessed: August 1, 2020: https://clinicaltrials.gov/ct2/show/NCT01362595.

39. Carrancio S, Markovics J, Wong P, et al.: An activin receptor IIA ligand trap promotes erythropoiesis resulting in a rapid induction of red blood cells and haemoglobin. Br J Haematol. 2014, 165:870-882. 10.1111/bjh. 12838

40. Vale W, Rivier J, Vaughan J, et al.: Purification and characterization of an FSH releasing protein from porcine ovarian follicular fluid. Nature.1986. 321:776-779. 10.1038/321776a0

41. Shiozaki M, Sakai R, Tabuchi M, Nakamura T, Sugino K, Sugino H, Eto Y: Evidence for the participation of endogenous activin A/erythroid differentiation factor in the regulation of erythropoiesis. Proc Natl Acad Sci U S A. 1992, 89:1553-1556. 10.1073/pnas.89.5.1553

42. Raftopoulos H, Laadem A, Hesketh PJ, et al.: Sotatercept (ACE-011) for the treatment of chemotherapyinduced anemia in patients with metastatic breast cancer or advanced or metastatic solid tumors treated with platinum-based chemotherapeutic regimens: results from two phase 2 studies. Support Care Cancer. 2016, 24:1517-1525. 10.1007/s00520-015-2929-9

43. Ear J, Huang H, Wilson T, et al.: RAP-011 improves erythropoiesis in zebrafish model of Diamond-Blackfan anemia through antagonizing lefty1. Blood. 2015, 126:880-890. 10.1182/blood-2015-01-622522

44. Safety and efficacy study of sotatercept in adults with transfusion dependent Diamond Blackfan anemia (ACE-011-DBA). Accessed: August 1, 2020: https://clinicaltrials.gov/ct2/show/NCT01464164.

45. Taylor AM, Raiser DM, Humphries JM, Ebert BL, Zon LI: Calmodulin inhibition rescues the effects of ribosomal protein deficiency by modulating p53 activity in models of Diamond Blackfan anemia. Blood. 2012, 120:512. 10.1182/blood.V120.21.512.512

46. Macari ER, Taylor A, Raiser D, et al.: Calmodulin inhibition rescues DBA models with ribosomal protein deficiency through reduction of RSK signaling. Blood. 2016, 128:332. 10.1182/blood.V128.22.332.332

47. Doulatov S, Vo LT, Macari ER, et al.: Drug discovery for Diamond-Blackfan anemia using reprogrammed hematopoietic progenitors. Sci Transl Med. 2017, 9:5645. 10.1126/scitranslmed.aah5645

48. Chai KY, Quijano CJ, Chiruka S: Danazol: an effective and underutilised treatment option in DiamondBlackfan anaemia. Case Rep Hematol. 2019, 2019:4684156. 10.1155/2019/4684156 